\title{
pRb and condensin-local control of global chromosome structure
}

\author{
Brigitte D. Lavoie ${ }^{1}$ \\ Department of Molecular Genetics, University of Toronto, Toronto, Ontario M5S 1A8, Canada
}

\begin{abstract}
Rb mutants exhibit aneuploidy and aberrant chromosome structure during mitosis. In this issue of Genes \& Development, a new paper from Longworth and colleagues (pp. 1011-1024) describes both physical and functional interactions between Drosophila Rbf1 and the dCAP-D3 subunit of condensin II. This work directly implicates the $\mathrm{Rb}$ family proteins in mitotic chromosome condensation and suggests that a failure in targeting condensin II to chromatin underlies the aneuploidy in rbf1 mutants.
\end{abstract}

\section{Discovery of condensins}

Over a hundred genomes have been sequenced to date, including the human genome in 2001, yet the mechanisms underlying eukaryotic genome transmission remain poorly defined. Genetic information is encoded within the long strands of DNA that make up chromosomes; however, the length and entanglement of interphase chromatin impose considerable challenges for chromosome segregation during mitosis. During replication, identical sister chromatids are first paired in order to define chromatids that must necessarily segregate away from each other if the genetic complement is to be maintained. Faithful partitioning, however, requires that in addition to pairing, the elongated and entangled chromatin of interphase be shortened and organized into discrete segregatable units: the condensed chromosomes. How chromosomes are condensed in mitosis remains a contentious and as-yet-unanswered question, with differences between experimental systems further highlighting the complexity of the problem (for recent reviews, see Hirano 2005a; Belmont 2006).

An important breakthrough toward a molecular understanding of higher-order chromosome structure was the identification in yeasts of the Structural Maintenance of Chromosomes (SMC) family of proteins (Strunnikov et al. 1993; Saka et al. 1994). SMC proteins are ubiquitous in nature and mediate diverse aspects of chromosome metabolism throughout the mitotic and meiotic cell cycles, including sister chromatid cohesion, mitotic

[Keywords: RBF1; RB; CAP-D3; chromatin; condensation; Condensin II] ${ }^{1}$ Correspondence.

E-MAIL brigitte.lavoie@utoronto.ca; FAX (416) 978-6885.

Article is online at http://www.genesdev.org/cgi/doi/10.1101/gad.1666808. chromosome condensation, DNA repair, and transcriptional regulation (for a recent review, see Hirano 2005b). A second breakthrough was the biochemical isolation of condensin, a five-subunit complex required for the in vitro condensation of DNA (Hirano et al. 1997) and in vivo segregation of chromosomes (Hagstrom and Meyer 2003 and references therein). While eukaryotic condensins are conserved from yeast to humans, two related condensin complexes, termed condensin I and II, have been identified in multicellular organisms (Table 1). Each complex comprises two core SMC proteins, a kleisin family protein and two HEAT repeat proteins, and while both condensins have been implicated in multiple aspects of chromosome metabolism, how each complex contributes to mitotic chromosome structure is poorly understood.

\section{In vitro activities of condensin}

Despite their conservation and requirement for life, little is known of the molecular functions of any of the condensin subunits. In vitro studies of the Smc2/4 heterodimer on naked DNA revealed multiple biochemical activities including ATPase hydrolysis, DNA-binding, renaturation, and knotting activities (Sutani and Yanagida 1997; Kimura and Hirano 2000; Stray et al. 2005). Studies using purified condensin holoenzyme uncovered two more activities: the ability to constrain positive supercoils and/or knots in the presence of topoisomerases (Kimura et al. 1997, 1999, 2001; Bazett-Jones et al. 2002). How these in vitro activities on naked DNA relate to mitotic chromosome condensation in the cell, however, remains to be established, since reactions using an $\sim 300$ times excess of condensin over that found in vivo $\quad(-30$ condensins per kilobase DNA in vitro vs. one condensin per $\sim 10 \mathrm{~kb}$ in vivo) (Sutani et al. 1999) are far from physiological. Furthermore, topoisomerase II activity, which is required for generating positively noded knots in vitro, is not necessary for mitotic chromosome condensation in budding yeast (Lavoie et al. 2002). While this could reflect the smaller size of budding yeast chromosomes, topoisomerase II knockdowns in mammalian and Drosophila cells also support significant levels of chromosome condensation (Chang et al. 2003; Carpenter and Porter 2004; Sakaguchi and Kikuchi 2004), arguing that 
Table 1. Subunits of condensin complexes in model organisms

\begin{tabular}{llllccc}
\hline Subunits & Human & Xenopus & Drosophila & C. elegans & $\begin{array}{c}\text { Saccharomyces } \\
\text { cerevisiae }\end{array}$ & $\begin{array}{c}\text { Schizosaccharomyces } \\
\text { pombe }\end{array}$ \\
\hline SMC & hCAP-E & XCAP-E & dSMC2 & MIX-1 & Smc2 & Cut14 \\
SMC & hCAP-C & XCAP-C & dSMC4 & SMC-4 & Smc4 & Cut3 \\
$\begin{array}{l}\text { Condensin I } \\
\text { Kleisin }\end{array}$ & hCAP-H & XCAP-H & Barren & - & Brn1 & Cnd2 \\
HEAT & hCAP-G & XCAP-G & dCAP-G & - & Ycg1 & Cnd3 \\
HEAT & hCAP-D2 & XCAP-D2 & dCAP-D2 & - & Ycs4 & Cnd1 \\
Condensin II & hCAP-H2 & XCAP-H2 & dCAP-H2 & KLE-2 & - & - \\
Kleisin & hCAP-G2 & XCAP-G2 & - & F55C5.4 & - & - \\
HEAT & hCAP-D3 & XCAP-D3 & dCAP-D3 & HCP-6 & - & - \\
\hline
\end{tabular}

condensin function does not depend absolutely on topoisomerase II in vivo.

\section{Mitotic functions of condensin}

A first step toward understanding the role of condensin in mitotic chromosome structure and function in vivo is important to clearly define benchmarks of the condensation process and identify those that are condensin-dependent. The inactivation and/or depletion of condensin activity from yeast to human cells results in chromatin bridges during anaphase, although the morphology of condensin-deficient metaphase chromosomes varies tremendously between experimental systems. For instance, in yeasts and Xenopus egg extracts where condensin I is the primary enzyme responsible for condensation, its inactivation/depletion abrogates mitotic chromosome folding, leaving metaphase chromatin in an interphaselike state (Saka et al. 1994; Hirano et al. 1997; Freeman et al. 2000; Lavoie et al. 2000; Ouspenski et al. 2000). In contrast, the inactivation of both condensin I and II (or condensin II alone in Caenorhabditis elegans) in the somatic cells of multicellular organisms supports a delayed but ultimately significant level of metaphase chromosome compaction, although it is important to stress that the resulting chromosomal structures withstand neither in vitro biochemical nor in vivo physical assaults (Steffensen et al. 2001; Hagstrom et al. 2002; Hudson et al. 2003; Gerlich et al. 2006a). The demonstration that additional and, presumably, condensin-independent activities function in correctly establishing and maintaining metaphase chromosome structure highlights the complexity of the overall reaction and the difficulties in deciphering overlapping functions in cells with multiple condensation activities. It seems reasonable to infer, however, that at least as far as condensins are concerned, condensin II, which is found on chromosomes throughout the cell cycle (as is condensin I in budding yeast), is likely to initiate condensation early in prophase, followed by condensin I function post-nuclear envelope breakdown (Ono et al. 2003, 2004; Gerlich et al. 2006a,b).

But how then do condensins promote mitotic chromosome folding in vivo? In multicellular organisms, the chromosomal sites at which each condensin complex functions remain to be determined. In contrast, model systems with a single condensin enzyme acting on a defined substrate provide substantial experimental advantages. The function of budding yeast condensin at the $\sim 1-\mathrm{Mb}$ repetitive rDNA array on chromosome XII has been characterized extensively, and provides a unique perspective since the enzymatic requirements for condensation are well-defined, both condensin and cohesin complex binding sites within the region have been mapped, and a step-wise chromosome assembly pathway has been proposed based on the identification of in vivo rDNA folding intermediates (Freeman et al. 2000; Lavoie et al. 2004; Wang et al. 2005; Johzuka et al. 2006).

Condensin is targeted to two sites within the rDNA nontranscribed spacers (NTS1 and NTS2) (Fig. 1) in interphase and mitosis (Freeman et al. 2000; Wang et al. 2005; Johzuka et al. 2006). Interestingly, no consensus binding sequence for condensin could be identified from genome-wide mapping experiments (Wang et al. 2005). Rather, the binding at the NTS1 is dependent on the replication fork block (RFB)-binding protein, Fob1, a sequence-specific DNA-binding protein, while binding at the NTS2 is affected by Pol I transcription (Johzuka et al. 2006; Wang et al. 2006; Johzuka and Horiuchi 2007). In addition to condensin, the cohesin complex, which functions in both pairing sister chromatids and mitotic chromosome condensation, also binds within the NTS, at the cohesin-associated region (CAR) (Fig. 1; Guacci et al. 1997; Laloraya et al. 2000).

While condensin and cohesin bind chromosomes independently of each other, both are required to organize the rDNA into a structure that can be segregated effectively. In cycling cells, the rDNA exists as a puff structure during interphase. As cells progress into mitosis, however, a number of condensin-dependent rDNA morphologies are observed by fluorescence in situ hybridization (Fig. 1B; Guacci et al. 1994; Lavoie et al. 2004). The repetitive nature of the rDNA amplifies small changes in chromosome morphology and/or stability, facilitating the visualization of partially condensed structures. The earliest condensed rDNA structures correspond to clusters, where the rDNA puff collapses into a more compact structure. Clusters subsequently resolve into progressively larger-though presumably more organizedforms, lines and metaphase loops; thus, the apparent size of the folding array is not an accurate indication of 


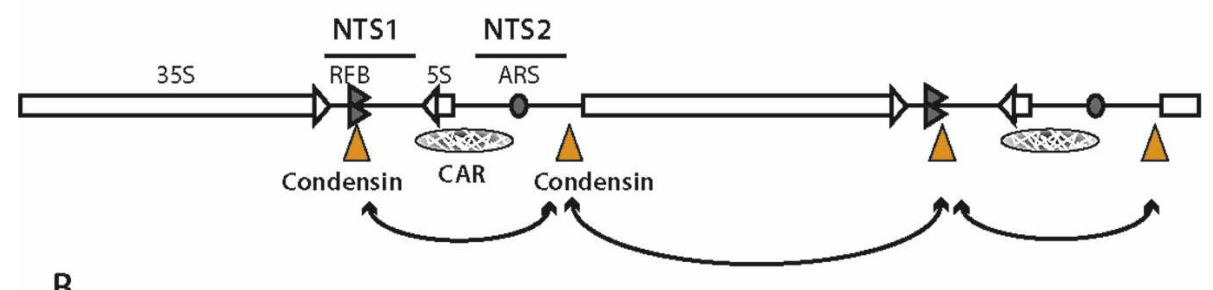

B

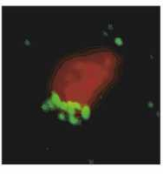

Cell cycle stage Interphase
rDNA morphology puff

Requirements

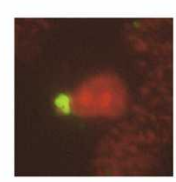

Prophase cluster

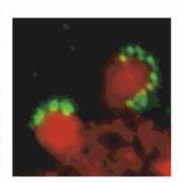

through line

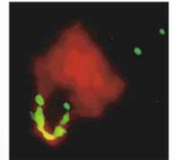

Metaphase loop

Condensin Cohesin

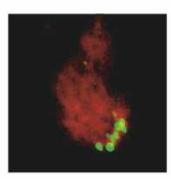

Anaphase hypercondensed

Condensin Aurora B/Ipl1 Cdc14

\section{C}

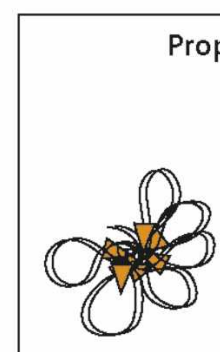

rDNA cluster

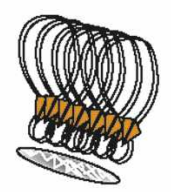

line

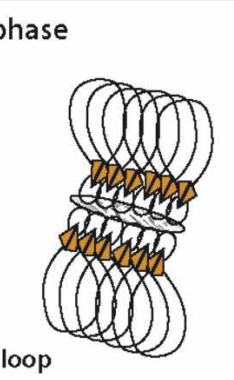

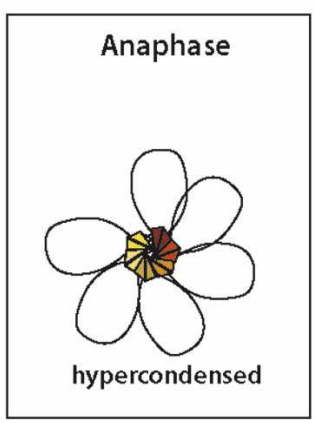

Figure 1. rDNA as a simple model for condensin-dependent chromosome organization. $(A)$ Schematic of the ribosomal DNA repeat. Each repeat comprises a 9.1-kb segment, encoding the 35S precursor rRNA and the 5S rRNA. Condensin (orange triangles) and cohesin (gray oval) complexes bind within NTS1 and NTS2. The RFB site, autonomous replicating sequence (ARS), and CAR are indicated. Arrows indicate potential interactions between condensin complexes bound at distinct sites. $(B)$ Multistep condensation pathways. Budding yeast cells were synchronized and processed for fluorescence in situ hybridization (Lavoie et al. 2004). The rDNA signal is shown in green with bulk chromosomes in red. In interphase, the $\sim 1-\mathrm{Mb}$ rDNA array adopts a disordered puff structure. As the cells progress through the cell cycle, the rDNA adopts a number of reproducible morphologies including cluster, line, and loop structures. Two condensin-dependent pathways orchestrate condensation: the metaphase condensation pathway and anaphase hypercondensation pathway. Requirements for each are indicated. $(C)$ Hypothetical model for higher-order rDNA folding. Condensin complexes (orange triangles) are targeted to NTS1 through the Fob1 protein, which directly binds to the RFB in each repeat. How condensin is directed to the Pol I promoter upstream of the 35S rRNA is unknown at present. Upon entry into mitosis, the cluster morphology would result from protein-protein interactions between condensin complexes. Cohesin complexes (gray ovals) serve to define domains within the rDNA array and facilitate the orderly interactions between condensins, which give rise to progressively more ordered structures. As the sister chromatids prepare for anaphase onset, they resolve into discrete segregatable units. In anaphase, cohesion between the sisters is dissolved and the Ipl1/AuroraB-dependent phosphorylation of condensin promote additional condensin-condensin interactions, leading to hypercondensation. The ordered packing of condensin complexes is depicted as triangles in graded colors. In higher eukaryotes, condensin II targeted to discrete sites by Rb family proteins could initiate a similar higher order folding during prophase, which would be further organized by condensin I-dependent interactions post-nuclear envelope breakdown.

the level of organization. While it is difficult to unequivocally equate morphologies with underlying mechanism, it is nonetheless important that hypothetical models of higher-order chromosome structure account for these partially condensed species, along with their enzymatic requirements. As a case in point, we showed that while morphologically identical rDNA species (clusters and short lines) could be observed both before and after anaphase onset, these species could none- theless be distinguished by their biochemical properties (Lavoie et al. 2004). Thus, even in the relatively simple system of the budding yeast rDNA, mitotic chromosome condensation is a multistep process that occurs through at least two distinct, cell cycle-regulated pathways: the metaphase pathway, which requires condensin and cohesin; and the anaphase hypercondensation pathway, which is dependent on condensin, the Aurora B/Ipll kinase, as well as the mitotic exit regulator, Cdc14 phos- 
phatase (D'Amours et al. 2004; Lavoie et al. 2004; Sullivan et al. 2004; Machin et al. 2005). Recently, the existence of a similar condensin and Aurora B-dependent hypercondensation pathway in mammalian anaphase cells has been reported (Mora-Bermudez et al. 2007), further supporting the validity of using the rDNA as a simple model for condensin function and extending it to more complex systems.

\section{Rbf1-a new player in mitotic chromosome condensation}

One of the challenges in deciphering the role of condensins in higher eukaryotes is that even the first steps of the condensation reaction (condensin binding) have been difficult to study. Since condensin does not recognize a defined DNA sequence motif-and given the technical limitations of ChIP-ChIP for mapping weak chromatin-binding sites in comparatively large genomes-it has not been straightforward to infer where condensins bind along chromosomes and, indeed, how the alternating stripes of condensin I and II complexes observed by immunofluorescence are derived (Ono et al. 2003). The study by Longworth et al. (2008) in this issue makes a significant contribution toward addressing this question. Longworth et al. (2008) identify an Rb family protein, Rbf1, that is required to target the condensin II subunit dCAPD3 to chromosomes in Drosophila and thus promotes chromosome condensation and faithful transmission.

$\mathrm{pRb}$ was identified $>20$ years ago as the first tumor suppressor, and its function as an important G1 cell cycle regulator has been characterized extensively (Macaluso et al. 2006). Less well understood, however, is why pRb mutants exhibit aneuploidy, a hallmark of most cancer cells. pRb has been shown to regulate the expression of the mitotic checkpoint protein Mad2, which promotes chromosome instability when misregulated in mammalian cells (Hernando et al. 2004; Sotillo et al. 2007). In Drosophila, however, mad2-null flies are viable and fertile, and do not exhibit dramatic chromosome instability (Buffin et al. 2007), suggesting that a distinct mechanism is likely to account for the chromosome segregation defects observed in rbf1 mutants.

In this issue, Longworth et al. (2008) investigate the nature of the mitotic defects in rbf1 mutants. Longworth et al. (2008) observed abnormally stretched chromosomes in rbf1 cells undergoing mitosis and, importantly, showed that this defect was functionally separable from Rbf1's roles in E2F transcriptional regulation. Rather, they showed that Rbf1 functions through the condensin II complex. Consistent with this, the activity of condensin II proved exquisitely sensitive to the level of Rbf 1 , since the overexpression of Rbf1 led to the hypercondensation of mitotic chromosomes and could be suppressed by reducing the level of dCAP-D3 in a heterozygous background. Strikingly, Longworth et al. (2008) further show that Drosophila Rbf1 interacts directly with dCAP-D3, and targets the subunit (and probably the entire condensin II complex) to chromosomes. The interaction between dCAP-D3 and Rbf1 also extended to the three human $\mathrm{Rb}$ family members, making a tantalizing argument that the functional interplay between $\mathrm{pRb}$ and condensin II will also be conserved.

The significance of this work stems from several aspects. First, a molecular understanding of mitotic chromosome condensation in vivo necessarily requires a fundamental understanding of the DNA-binding preferences and requirements of the two condensin complexes. Condensin I was known previously to be targeted to chromosomes through the A kinase-anchoring protein AKAP95 (Steen et al. 2000; Eide et al. 2002). Longworth et al. (2008) now provide the first insight into how condensin II targeting is achieved, and demonstrate that Rbfl associates specifically with condensin II and not condensin I. This is an important step forward and will undoubtedly facilitate finding the DNA sites where condensin II initiates condensation. Second, Longworth et al. (2008) provide a simple and elegant model for the condensation defects and aneuploidy observed in $r b f 1$ mutant animals. While it is likely that Rbf1 functions by directing condensin II function at specific loci, it remains a formal possibility that its effects on aneuploidy could be mediated solely through the dCAP-D3 subunit. Finally, this work strongly suggests that even subtle disruptions of condensin function during mitosis may have profound biological consequences. Consistent with this, mutations in the SMC2 and SMC4 subunits were found in cases of pyothorax-associated lymphoma (Ham et al. 2007), and loss of heterozygosity for the region encompassing hCAP-D3 is associated with breast cancer (Gentile et al. 2001).

\section{Condensin-a new player in $\mathbf{R b}$ protein function?}

Beyond their role in mitosis, condensins also function in interphase, notably in transcriptional regulation and gene silencing. In yeasts, condensin modulates heterochromatin function both positively and negatively, in a locus-specific manner (Bhalla et al. 2002; Machin et al. 2004; Chen et al. 2008). Similarly, condensin has been implicated in heterochromatin maintenance in Drosophila as dCAP-G, a component of both condensin complexes, has been reported to both enhance and suppress position effect variegation (PEV) (Dej et al. 2004; Cobbe et al. 2006) while dCAP-D3 mutants suppress PEV (Longworth et al. 2008). Consistent with a role for condensin in heterochromatin function/transcriptional regulation, condensin II subunits have also been implicated in cellular differentiation, notably during erythroid cell development (murine CAP-G2) and T-cell differentiation (kleisin $\beta$ ) (Xu et al. 2006; Gosling et al. 2007). Within this context, it is tempting to speculate that the functional interplay between condensin II and $\mathrm{Rb}$ family members could be reciprocated and that the nuclear localization of condensin II throughout the cell cycle reflects this. Regardless of whether or not this proves to be the case, the emerging links between $\mathrm{pRb}$, condensin II, and cancer are likely to provide fresh insight into multiple aspects of chromosome metabolism and the maintenance of genomic integrity. 


\section{Acknowledgments}

I am funded by CIHR operating (MOP57913) and salary (MSH63646) grants.

\section{References}

Bazett-Jones, D.P., Kimura, K., and Hirano, T. 2002. Efficient supercoiling of DNA by a single condensin complex as revealed by electron spectroscopic imaging. Mol. Cell 9: 11831190.

Belmont, A.S. 2006. Mitotic chromosome structure and condensation. Curr. Opin. Cell Biol. 18: 632-638.

Bhalla, N., Biggins, S., and Murray, A.W. 2002. Mutation of YCS4, a budding yeast condensin subunit, affects mitotic and nonmitotic chromosome behavior. Mol. Biol. Cell 13: 632-645.

Buffin, E., Emre, D., and Karess, R.E. 2007. Flies without a spindle checkpoint. Nat. Cell Biol. 9: 565-572.

Carpenter, A.J. and Porter, A.C. 2004. Construction, characterization, and complementation of a conditional-lethal DNA topoisomerase II $\alpha$ mutant human cell line. Mol. Biol. Cell 15: 5700-5711.

Chang, C.J., Goulding, S., Earnshaw, W.C., and Carmena, M. 2003. RNAi analysis reveals an unexpected role for topoisomerase II in chromosome arm congression to a metaphase plate. J. Cell Sci. 116: 4715-4726.

Chen, E.S., Zhang, K., Nicolas, E., Cam, H.P., Zofall, M., and Grewal, S.I. 2008. Cell cycle control of centromeric repeat transcription and heterochromatin assembly. Nature 451: 734-737.

Cobbe, N., Savvidou, E., and Heck, M.M. 2006. Diverse mitotic and interphase functions of condensins in Drosophila. Genetics 172: 991-1008.

D'Amours, D., Stegmeier, F., and Amon, A. 2004. Cdc14 and condensin control the dissolution of cohesin-independent chromosome linkages at repeated DNA. Cell 117: 455-469.

Dej, K.J., Ahn, C., and Orr-Weaver, T.L. 2004. Mutations in the Drosophila condensin subunit dCAP-G: Defining the role of condensin for chromosome condensation in mitosis and gene expression in interphase. Genetics 168: 895-906.

Eide, T., Carlson, C., Tasken, K.A., Hirano, T., Tasken, K., and Collas, P. 2002. Distinct but overlapping domains of AKAP95 are implicated in chromosome condensation and condensin targeting. EMBO Rep. 3: 426-432.

Freeman, L., Aragon-Alcaide, L., and Strunnikov, A. 2000. The condensin complex governs chromosome condensation and mitotic transmission of rDNA. J. Cell Biol. 149: 811-824.

Gentile, M., Wiman, A., Thorstenson, S., Loman, N., Borg, A., and Wingren, S. 2001. Deletion mapping of chromosome segment 11q24-q25, exhibiting extensive allelic loss in early onset breast cancer. Int. J. Cancer 92: 208-213.

Gerlich, D., Hirota, T., Koch, B., Peters, J.M., and Ellenberg, J. 2006a. Condensin I stabilizes chromosomes mechanically through a dynamic interaction in live cells. Curr. Biol. 16: 333-344.

Gerlich, D., Koch, B., Dupeux, F., Peters, J.M., and Ellenberg, J. 2006b. Live-cell imaging reveals a stable cohesin-chromatin interaction after but not before DNA replication. Curr. Biol. 16: $1571-1578$.

Gosling, K.M., Makaroff, L.E., Theodoratos, A., Kim, Y.H., Whittle, B., Rui, L., Wu, H., Hong, N.A., Kennedy, G.C., Fritz, J.A., et al. 2007. A mutation in a chromosome condensin II subunit, kleisin $\beta$, specifically disrupts T cell development. Proc. Natl. Acad. Sci. 104: 12445-12450.
Guacci, V., Hogan, E., and Koshland, D. 1994. Chromosome condensation and sister chromatid pairing in budding yeast. J. Cell Biol. 125: 517-530.

Guacci, V., Koshland, D., and Strunnikov, A. 1997. A direct link between sister chromatid cohesion and chromosome condensation revealed through the analysis of MCD1 in S. cerevisiae. Cell 91: 47-57.

Hagstrom, K.A. and Meyer, B.J. 2003. Condensin and cohesin: More than chromosome compactor and glue. Nat. Rev. Genet. 4: 520-534.

Hagstrom, K.A., Holmes, V.F., Cozzarelli, N.R., and Meyer, B.J. 2002. C. elegans condensin promotes mitotic chromosome architecture, centromere organization, and sister chromatid segregation during mitosis and meiosis. Genes \& Dev. 16: 729-742.

Ham, M.F., Takakuwa, T., Rahadiani, N., Tresnasari, K., Nakajima, H., and Aozasa, K. 2007. Condensin mutations and abnormal chromosomal structures in pyothorax-associated lymphoma. Cancer Sci. 98: 1041-1047.

Hernando, E., Nahle, Z., Juan, G., Diaz-Rodriguez, E., Alaminos, M., Hemann, M., Michel, L., Mittal, V., Gerald, W., Benezra, R., et al. 2004. Rb inactivation promotes genomic instability by uncoupling cell cycle progression from mitotic control. Nature 430: 797-802.

Hirano, T. 2005a. Condensins: Organizing and segregating the genome. Curr. Biol. 15: R265-R275. doi: 10.1016/ j.cub.2005.03.037.

Hirano, T. 2005b. SMC proteins and chromosome mechanics: From bacteria to humans. Philos. Trans. R. Soc. Lond. B Biol. Sci. 360: 507-514.

Hirano, T., Kobayashi, R., and Hirano, M. 1997. Condensins, chromosome condensation protein complexes containing XCAP-C, XCAP-E and a Xenopus homolog of the Drosophila Barren protein. Cell 89: 511-521.

Hudson, D.F., Vagnarelli, P., Gassmann, R., and Earnshaw, W.C. 2003. Condensin is required for nonhistone protein assembly and structural integrity of vertebrate mitotic chromosomes. Dev. Cell 5: 323-336.

Johzuka, K. and Horiuchi, T. 2007. RNA polymerase I transcription obstructs condensin association with 35S rRNA coding regions and can cause contraction of long repeat in Saccharomyces cerevisiae. Genes Cells 12: 759-771.

Johzuka, K., Terasawa, M., Ogawa, H., Ogawa, T., and Horiuchi, T. 2006. Condensin loaded onto the replication fork barrier site in the rRNA gene repeats during $S$ phase in a FOB1dependent fashion to prevent contraction of a long repetitive array in Saccharomyces cerevisiae. Mol. Cell. Biol. 26: 22262236.

Kimura, K. and Hirano, T. 2000. Dual roles of the 11 S regulatory subcomplex in condensin functions. Proc. Natl. Acad. Sci. 97: 11972-11977.

Kimura, M., Kotani, S., Hattori, T., Sumi, N., Yoshioka, T., Todokoro, K., and Okano, Y. 1997. Cell cycle-dependent expression and spindle pole localization of a novel human protein kinase, Aik, related to Aurora of Drosophila and yeast Ipl1. J. Biol. Chem. 272: 13766-13771.

Kimura, K., Rybenkov, V.V., Crisona, N.J., Hirano, T., and Cozzarelli, N.R. 1999. 13 S condensin actively reconfigures DNA by introducing global positive writhe: Implications for chromosome condensation. Cell 98: 239-248.

Kimura, K., Cuvier, O., and Hirano, T. 2001. Chromosome condensation by a human condensin complex in Xenopus egg extracts. J. Biol. Chem. 276: 5417-5420.

Laloraya, S., Guacci, V., and Koshland, D. 2000. Chromosomal addresses of the cohesin component Mcd1p. J. Cell Biol. 151: 1047-1056. 
Lavoie, B.D., Tuffo, K.M., Oh, S., Koshland, D., and Holm, C. 2000. Mitotic chromosome condensation requires Brn1p, the yeast homologue of Barren. Mol. Biol. Cell 11: 1293-1304.

Lavoie, B.D., Hogan, E., and Koshland, D. 2002. In vivo dissection of the chromosome condensation machinery: Reversibility of condensation distinguishes contributions of condensin and cohesin. J. Cell Biol. 156: 805-815.

Lavoie, B.D., Hogan, E., and Koshland, D. 2004. In vivo requirements for rDNA chromosome condensation reveal two cellcycle-regulated pathways for mitotic chromosome folding. Genes \& Dev. 18: 76-87.

Longworth, M.S., Herr, A., Ji, J.-Y., and Dyson, N.J. 2008. RBF1 promotes chromatin condensation through a conserved interaction with the Condensin II protein, dCAP-D3. Genes \& Dev. (this issue), doi: 10.1101/gad.1631508.

Macaluso, M., Montanari, M., and Giordano, A. 2006. Rb family proteins as modulators of gene expression and new aspects regarding the interaction with chromatin remodeling enzymes. Oncogene 25: 5263-5267.

Machin, F., Paschos, K., Jarmuz, A., Torres-Rosell, J., Pade, C., and Aragon, L. 2004. Condensin regulates rDNA silencing by modulating nucleolar Sir2p. Curr. Biol. 14: 125-130.

Machin, F., Torres-Rosell, J., Jarmuz, A., and Aragon, L. 2005. Spindle-independent condensation-mediated segregation of yeast ribosomal DNA in late anaphase. J. Cell Biol. 168: 209-219.

Mora-Bermudez, F., Gerlich, D., and Ellenberg, J. 2007. Maximal chromosome compaction occurs by axial shortening in anaphase and depends on Aurora kinase. Nat. Cell Biol. 9: 822-831.

Ono, T., Losada, A., Hirano, M., Myers, M.P., Neuwald, A.F., and Hirano, T. 2003. Differential contributions of condensin I and condensin II to mitotic chromosome architecture in vertebrate cells. Cell 115: 109-121.

Ono, T., Fang, Y., Spector, D.L., and Hirano, T. 2004. Spatial and temporal regulation of Condensins I and II in mitotic chromosome assembly in human cells. Mol. Biol. Cell 15: 32963308.

Ouspenski, I.I., Cabello, O.A., and Brinkley, B.R. 2000. Chromosome condensation factor Brn $1 \mathrm{p}$ is required for chromatid separation in mitosis. Mol. Biol. Cell 11: 1305-1313.

Saka, Y., Sutani, T., Yamashita, Y., Saitoh, S., Takeuchi, M., Nakaseko, Y., and Yanagida, M. 1994. Fission yeast cut3 and cut14, members of the ubiquitous protein family, are required for chromosome condensation and segregation in mitosis. EMBO I. 13: 4938-4952.

Sakaguchi, A. and Kikuchi, A. 2004. Functional compatibility between isoform $\alpha$ and $\beta$ of type II DNA topoisomerase. $J$. Cell Sci. 117: 1047-1054.

Sotillo, R., Hernando, E., Diaz-Rodriguez, E., Teruya-Feldstein, J., Cordon-Cardo, C., Lowe, S.W., and Benezra, R. 2007. Mad2 overexpression promotes aneuploidy and tumorigenesis in mice. Cancer Cell 11: 9-23.

Steen, R.L., Cubizolles, F., Le Guellec, K., and Collas, P. 2000. A kinase-anchoring protein (AKAP) 95 recruits human chromosome-associated protein (hCAP)-D2/Eg7 for chromosome condensation in mitotic extract. J. Cell Biol. 149: 531-536.

Steffensen, S., Coelho, P.A., Cobbe, N., Vass, S., Costa, M., Hassan, B., Prokopenko, S.N., Bellen, H., Heck, M.M., and Sunkel, C.E. 2001. A role for Drosophila SMC4 in the resolution of sister chromatids in mitosis. Curr. Biol. 11: 295307.

Stray, J.E., Crisona, N.J., Belotserkovskii, B.P., Lindsley, J.E., and Cozzarelli, N.R. 2005. The Saccharomyces cerevisiae SMC2/4 condensin compacts DNA into $(+)$ chiral structures without net supercoiling. J. Biol. Chem. 280: 34723-34734.
Strunnikov, A.V., Larionov, V.L., and Koshland, D. 1993. SMC1: An essential yeast gene encoding a putative head-rod-tail protein is required for nuclear division and defines a new ubiquitous protein family. J. Cell Biol. 123: 1635-1648.

Sullivan, M., Higuchi, T., Katis, V.L., and Uhlmann, F. 2004. Cdc14 phosphatase induces rDNA condensation and resolves cohesin-independent cohesion during budding yeast anaphase. Cell 117: 471-482.

Sutani, T. and Yanagida, M. 1997. DNA renaturation activity of the SMC complex implicated in chromosome condensation. Nature 388: 798-801.

Sutani, T., Yuasa, T., Tomonaga, T., Dohmae, N., Takio, K., and Yanagida, M. 1999. Fission yeast condensin complex: Essential roles of non-SMC subunits for condensation and Cdc2 phosphorylation of Cut3/SMC4. Genes \& Dev. 13: 22712283.

Wang, B.D., Eyre, D., Basrai, M., Lichten, M., and Strunnikov, A.V. 2005. Condensin binding at distinct and specific chromosomal sites in the Saccharomyces cerevisiae genome. Mol. Cell. Biol. 25: 7216-7225.

Wang, B.D., Butylin, P., and Strunnikov, A. 2006. Condensin function in mitotic nucleolar segregation is regulated by rDNA transcription. Cell Cycle 5: 2260-2267.

$\mathrm{Xu}$, Y., Leung, C.G., Lee, D.C., Kennedy, B.K., and Crispino, J.D. 2006. MTB, the murine homolog of condensin II subunit CAP-G2, represses transcription and promotes erythroid cell differentiation. Leukemia 20: 1261-1269. 


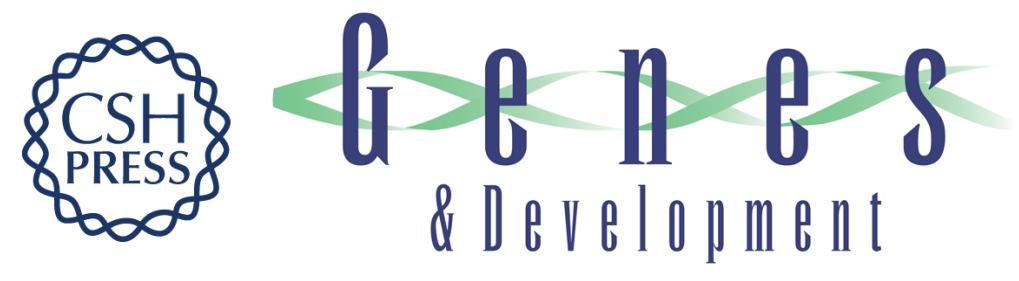

\section{pRb and condensin--local control of global chromosome structure}

Brigitte D. Lavoie

Genes Dev. 2008, 22:

Access the most recent version at doi:10.1101/gad.1666808

Related Content RBF1 promotes chromatin condensation through a conserved interaction with the Condensin II protein dCAP-D3

Michelle S. Longworth, Anabel Herr, Jun-Yuan Ji, et al.

Genes Dev. April , 2008 22: 1011-1024

References This article cites 57 articles, 27 of which can be accessed free at: http://genesdev.cshlp.org/content/22/8/964.full.html\#ref-list-1

Articles cited in:

http://genesdev.cshlp.org/content/22/8/964.full.html\#related-urls

\section{License}

Email Alerting

Receive free email alerts when new articles cite this article - sign up in the box at the top

Service right corner of the article or click here.

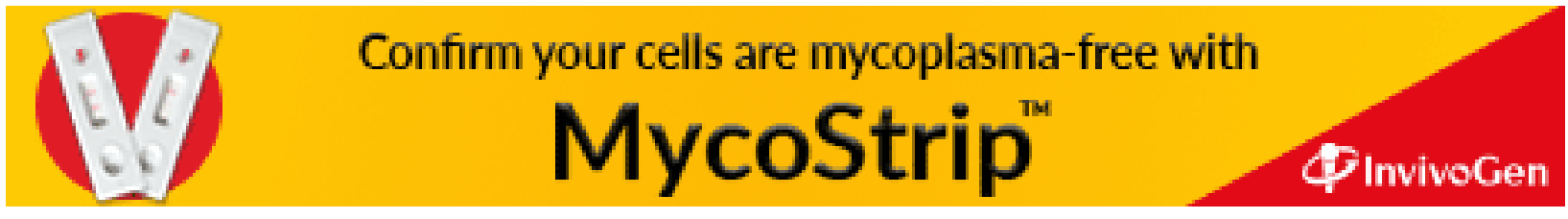

